\title{
Relación del autoconcepto físico con las conductas de consumo de alcohol y tabaco en adolescentes
}

\section{Relationship between physical self-concept and alcohol and tobacco use behaviours in adolescents}

\author{
Juan Antonio Moreno'; Remedios Moreno²; Eduardo \\ Cervelló ${ }^{3}$
}

\author{
${ }^{1}$ Universidad Miguel Hernández de Elche \\ 2 Unidad de Investigación en Educación Física y Deportes \\ ${ }^{3}$ Universidad Miguel Hernández de Elche \\ Enviar correspondencia a: \\ Juan Antonio Moreno Murcia. Departamento de Psicología de la \\ Salud. Universidad Miguel Hernández de Elche. Edificio Torrevaillo. \\ Avenida de la Universidad, s/n. 03202 Elche (Alicante) España. \\ E-mail: j.moreno@umh.es. Tel: +34 965222046
}

\section{RESUMEN}

El propósito de este estudio ha sido comprobar la relación del género, el consumo de tabaco y alcohol y la práctica físico-deportiva en el autoconcepto físico en estudiantes adolescentes españoles de educación física. La muestra estuvo compuesta por 1.008 alumnos ( $\mathrm{M}$ edad $=15.94$, $D T=.89)$ a los que se les pasó el cuestionario de autoconcepto físico (PSO). Tras distintos análisis de correlación, univariantes, multivariantes y de regresión, los resultados revelaron que el autoconcepto físico presentaba relaciones con el género, el consumo de tabaco y alcohol y la práctica físico-deportiva. La mejor percepción de la imagen corporal se observó en las mujeres que no fumaban y los no practicantes de actividad físico-deportiva. Por otro lado, el ser varón y practicar deporte fue lo que más predecía el autoconcepto físico, entendiendo por este la autovaloración física. Como conclusión, este estudio muestra la relevancia que tiene el resultado de que la acción de fumar se asocia con una buena imagen corporal y que la práctica del deporte se realiza para fomentar una apariencia física más positiva y acorde con los cánones de belleza establecidos en nuestra sociedad actual.

Palabras clave: Autoconcepto físico, género, deporte, educación física, alcohol, tabaco.

\section{ABSTRACT}

The purpose of this study was to analyze the effects of gender, alcohol use, smoking behaviour and the practice of sport on physical selfconcept among Spanish students of physical education. A total of 1.008 students ( $M$ age $=15.94, S D=89$ ) participated in the study, and completed the physical self-concept questionnaire (PSO). The results revealed that physical self-concept was related to gender, alcohol use, smoking behaviour and the practice of sport. The best perception of body image was found in women who did not smoke and participants (male and female) who did not do sport. On the other hand, being male and practicing sport predicted general physical self-concept. This study shows the relevance of the belief that smoking is associated with good body image and that sport is practiced to improve physical appearance.

Key words: Physical self-concept, age, gender, sport, physical education, tobacco, alcohol. 


\section{INTRODUCCIÓN}

Numerosas investigaciones han constatado los efectos beneficiosos que la práctica de actividad física adecuada, regular y controlada posee para la salud y calidad de vida (Colbert, Hartman, Tangrea, Pietinen, Virtamo, Taylor et al., 2002; Shepard, 1995; Teixeira, Sardinha, Going, y Lohman, 2001). Por tanto, una práctica de actividad física-deportiva realizada bajo unos determinados parámetros de frecuencia, intensidad y duración está encuadrada dentro de los llamados modelos o estilos de vida saludables. La promoción de la práctica de actividad física y deportiva se ha convertido en uno de los objetivos esenciales en la política educativa de la mayoria de paises desarrollados.

Por otra parte, también es abundante la investigación que señala los perjuicios para la salud del consumo de tabaco y alcohol según autores como Bernaards, Twisk, Van Mechelen, Snel y Kemper (2003), Melnick, Miller, Sabo, Farrell y Barnes (2001) y Packa, Massamba, Mabiala, Oniangue, Bahanguila, Gnama et al. (2002), asociándose la práctica regular de ejercicio físico a la reducción de conductas de consumo de tabaco (Bergamaschi, Morri, Resi, Zanetti, y Stampi, 2002; Holmen, Barrett, Clausen, Holemn, y Bjermer, 2002; Nistal, Prieto, Del Valle, y González, 2003). En este sentido, la proliferación en los últimos años del consumo de tabaco y alcohol entre los jóvenes ha despertado una gran alarma social mostrando diferencias en el consumo del primero atendiendo al género y la edad de los adolescentes (Mendoza y López Pérez, 2007). Las diferentes investigaciones sobre las conductas sociales de consumo durante la adolescencia confirman el aumento paulatino en la instauración y desarrollo del consumo de substancias tóxicas, que se han consolidado progresivamente como hábitos de vida en los adolescentes (Tercedor, Martin-Matillas, Chillón, Pérez López, Ortega, Warnberg et al., 2007). Esta instauración del hábito tabáquico puede deberse en gran medida a la influencia que ejercen los adolescentes como medio de inclusión en diferentes grupos sociales existentes en la actualidad (Calleja y Aguilar, 2008).

Es conocido que la práctica de una actividad física regular, estable y moderada ayuda a mejorar tanto la salud física como la psicológica. En relación a esta última dimensión, la idea de cuerpo asociada a la imagen corporal y el fuerte arraigo de culto al cuerpo en la actual sociedad, ha sido (Smith, Handley y Eldredge, 1998) y está siendo (Lau, Cheung, y Ransdell, 2008; Perry et al., 2002) objeto de estudio. Ambos aspectos, el cuerpo y la apariencia, se reflejan como el predictor más influyente en la autoestima (Fox, 1997; Harter, 1993), quedando todas esas dimensiones incluidas en el constructo del autoconcepto físico.

Basándose en el modelo del autoconcepto multidimensional de Shavelson, Hubner y Stanton, (1976) y de Fox y Corbin (1989) desarrollaron el Perfil de Auto-percepción Físico (PSPP) dividiendo el autoconcepto físico en cuatro subdominios (la competencia física percibida, la fuerza física, la condición física y el atractivo físico, apariencia o imagen corporal) junto con un quinto y más general (la autoestima). La percepción de la condición física se ha manifestado como el predictor más fuerte en la conducta del ejercicio físico (Fox y Corbin, 1989; Sonströem, Speliotis, y Fava, 1992) atribuyendo diferentes valores (mejor o peor) al aspecto físico destacando la importancia de la competencia física percibida. Resaltando a su vez que esta afecta a la conducta del ejercicio y a la auto-evaluación física del individuo (Harter, 1986). Los efectos positivos en la autoestima y el autoconcepto físico, también se han atribuido a la práctica físico-deportiva (Bruya, 1977; McGowan, Jarman, y Pedersen, 1974).

Respecto al género, numerosos estudios demuestran que las mujeres tienen mayor preocupación por su cuerpo y su imagen (Bane y McAuley, 1998), mostrando que estas son más críticas con sus cuerpos y están más preocupadas por la apariencia física que los hombres (De Gracia, Marcó, y Trujado, 2007; Heunnmann, Shapiro, Hampton, y Mitchell, 1966; Loland, 1998). Del mismo modo, las mujeres presentan menores puntuaciones en todos los subdominios del autoconcepto físico arriba citados respecto a los varones (Fox y Corbin, 1989; Hayes, Crocker y Kowalski 1999) y también parecen asociar más el atractivo corporal con un mejor autoconcepto físico general. Mientras que los hombres se basan más en el deporte y aspectos de fuerza física en relación con el ego personal (Asçi, Asçi, y Zorba, 1999; Hayes et al., 1999; Moreno, Cervelló, y Moreno, 2008).

Teniendo en cuenta los resultados obtenidos por estudios previos (Lindwall y Hassmén, 2004; Maïano, Ninot, y Bilard, 2004; Moreno y Cervelló, 2005; Moreno et al., 2008) en este trabajo se hipotetiza que los chicos presentarán un mejor autoconcepto físico que las chicas, mientras que aquellos que fuman y beben presentarán un peor autoconcepto físico. También se espera encontrar mejores niveles de autoconcepto físico en los practicantes de actividades físicas deportivas extraescolares. Así pues, el objetivo de este estudio ha sido comprobar las relaciones del género, el consumo de tabaco y alcohol y la práctica físico-deportiva en el autoconcepto físico en adolescentes en contraposición con los estudios realizados por Jiménez, Musitu y Murgui (2008) que muestran escasa relación entre estos aspectos.

\section{MÉTODO}

\section{Participantes}

La muestra estuvo compuesta por 1.008 estudiantes de Educación Secundaria (442 varones y 566 mujeres), de edades comprendidas entre los 15 y 17 años $(M=15.94, D T=$ $0.89)$. Del total de la muestra, el $39.1 \%(n=394)$ no realizaba ninguna práctica físico-deportiva, mientras que el 60.9\% $(n=614)$ sí que practicaba extraescolarmente. 190 sujetos consumían tabaco frente a los 818 que no, mientras que el $50 \%$ aproximadamente $(n=506)$ consumían alcohol frente al $50 \%$ restante $(n=502)$ que no lo hacia. 


\section{Instrumentos}

Cuestionario de Autoconcepto físico (PSO). La adaptación al español de esta escala (Moreno y Cervelló, 2005) partió de la original Physical Self-Perception Profile (PSPP) de Fox (1990) y Fox y Corbin (1989). El instrumento estaba compuesto por 30 ítems y cinco factores: competencia percibida, imagen corporal, condición física, fuerza física y autoestima. Las respuestas al instrumento estaban expresadas en una escala tipo Lickert, en la que 1 corresponde a totalmente en desacuerdo y el 4 a totalmente de acuerdo. En nuestro estudio los valores "alfas" de Cronbach obtenidos fueron de 0.68 para la fuerza, 0.76 para la competencia percibida, 0.87 para la condición física, 0.81 para la imagen corporal y 0.77 para la autoestima. El factor fuerza física mostró un valor alfa inferior al recomendado 0.80 (Nunnally, 1978), pero según Hair et al. (1998), la validez interna observada puede ser marginalmente aceptada al estar compuesto por pocos items.

Práctica físico-deportiva extraescolar, consumo de alcohol y tabaco. A los practicantes se les preguntó a través de un solo ítem si practicaban deporte y si consumian alcohol y tabaco, de forma que únicamente existían dos posibilidades de respuesta "síno". A los practicantes se les preguntó a través de dos ítems si practicaban deporte existiendo dos posibilidades de respuesta "no/si" especificando en caso positivo "¿qué deporte practicas?", "¿Cuánto tiempo practicas diariamente?" expresado en minutos, "¿Cuántos días practicas?" relacionando las respuestas 1 "puntualmente", 2 "dos o tres dias a la semana" y 3 "más de tres días a la semana". Continuando con la última pregunta al respecto "¿Cuánto tiempo llevas practicando?" con opciones de respuesta múltiple como 1 "menos de un año", 2 "entre uno y tres años"y 3 "más de tres años". Para el consumo de alcohol se respondia con dos posibilidades 1 "no" y 2 "si" especificando esta opción "¿Cuántas copas?" con posibles respuestas como 1 "Alguna en la semana", 2 "una o dos al dia" y 3 "tres o más al día". Por último el consumo de tabaco viene medido por la pregunta "¿fumas?" con opción de respuesta "no" y "si" matizando en este caso "¿Cuántos cigarrillos?" donde 1 equivale a "de cero a cinco al día", 2 "de cinco a diez al día" y 3 "más de diez al día".

\section{Procedimiento}

Se llevó a cabo una selección de centros atendiendo a un muestreo aleatorio por conglomerados (Azorín y SánchezCrespo, 1986). La cumplimentación del cuestionario se realizó en el aula de educación física, no estando presente el profesor pero sí el investigador principal con el fin de solventar toda duda que pudiera surgir en la comprensión tanto de los ítems que componían el cuestionario como de las instrucciones establecidas al inicio de la administración del mismo. Las instrucciones establecidas previas a la cumplimentación del cuestionario hicieron referencia al objetivo general del estudio, con el fin de que mostraran mayor interés en la mecánica de rellenado del mismo. Del mismo modo, se aclararon algunos términos que pudieran resultar confusos y se les animó para que lo rellenaran de la manera más sincera posible haciendo hincapié en el anonimato de los mismos. Por ser menores de edad se solicitó una autorización paterna para su participación en el estudio. En todos los casos se indicó que la participación en la investigación era voluntaria. El tiempo requerido para la cumplimentación del cuestionario fue de 10-15 minutos en función del número de alumnos y la agilidad de la clase.

\section{Análisis de datos}

Se ha realizado un análisis descriptivo de las variables utilizadas en el estudio. Para analizar la consistencia interna del instrumento utilizado en la investigación se utilizó el coeficiente alfa de Cronbach. Las relaciones entre variables se exploraron utilizando correlaciones bivariadas de Pearson, mientras que las diferencias en autoconcepto físico relativas al género, consumo de alcohol y tabaco, y práctica físico-deportiva extraescolar fueron obtenidas utilizando un MANOVA. Por último, para comprobar el poder de predicción del género, consumo de tabaco y alcohol y práctica físicodeportiva extraescolar en el autoconcepto físico se utilizó un análisis de regresión lineal. Se utilizó el programa SPSS en su versión 15.0 para el análisis de los datos.

\section{RESULTADOS}

\section{Medias, desviaciones típicas y análisis de correlación}

Para la globalidad de la muestra, respecto a la relación de las medias se observó que el mayor valor correspondió al factor autoestima, seguida de la condición física, continuando con la competencia física y fuerza y por último, la imagen corporal. En relación a la correlación de los factores del autoconcepto físico, todos se correlacionaban positiva y significativamente con todos, exceptuando la dimensión imagen corporal que lo hacía de forma negativa (Tabla 1).

En relación a la dirección de las correlaciones respecto al género (Tabla 4), se encontraron correlaciones en todas las dimensiones, siendo los chicos quienes tenían una mayor autopercepción en todos los subdominios frente a las chicas de manera que los chicos puntuaron más alto en las dimensiones condición física, competencia percibida y fuerza, mientras que las chicas lo realizaron en la dimensión imagen corporal. Con respecto a la conducta de consumo de tabaco se han obtenido correlaciones significativas, aunque muy bajas, en los factores condición física, imagen corporal, competencia percibida y fuerza. Para la variante práctica deportiva se han encontrado correlaciones significativas a favor de los que practicaban, en las dimensiones condición física, competencia percibida, fuerza y autoestima, mientras que los que no practicaban lo han hecho en el factor imagen corporal, aunque con una correlación muy baja. No se han encontrado correlaciones entre la conducta de consumo de alcohol y el autoconcepto físico. 
Tabla 1

Media, Desviación Estándar, Coeficiente Alfa y Correlaciones de Todas las Variables.

\begin{tabular}{lcccccccc}
\hline & $M$ & $D T$ & $\alpha$ & 1 & 2 & 3 & 4 & 5 \\
\hline $\begin{array}{l}\text { 1. Condición } \\
\text { fisica }\end{array}$ & 2.58 & .96 & .87 & - & $-.74^{* *}$ & $.79^{* *}$ & $.74^{* *}$ & $.78^{* *}$ \\
$\begin{array}{l}\text { 2. Imagen } \\
\text { corporal }\end{array}$ & 2.34 & .77 & .81 & - & - & $-.68^{* *}$ & $-.61^{* *}$ & $-.67^{* *}$ \\
$\begin{array}{l}\text { 3. Competencia } \\
\text { percibida }\end{array}$ & 2.44 & .83 & .76 & - & - & - & $.73^{* *}$ & $.73^{* *}$ \\
$\begin{array}{l}\text { 4. Fuerza } \\
\text { 5. Autoestima }\end{array}$ & 2.37 & .83 & .68 & - & - & - & - & $.69^{* *}$ \\
& 2.68 & .87 & .77 & - & - & - & - & -
\end{tabular}

${ }^{* *} p<.001$

Relaciones principales y relaciones de interacción del género, consumo de tabaco y alcohol y la práctica físico-deportiva sobre el autoconcepto físico

Se realizó un análisis multivariante (Tablas 2 y 3 ) donde se consideraron como variables independientes el género, consumo de tabaco y alcohol y práctica físico-deportiva extraescolar y como variables dependientes la condición físi$\mathrm{ca}$, imagen corporal, competencia percibida, fuerza y autoestima. El resultado de los contrastes multivariados mostró una relación de interacción entre el consumo de alcohol y la práctica deportiva confirmando que los consumidores rechazaban la práctica de actividad físico-deportiva alegando su buena forma física sin necesidad de practicar (Lambda de Wilks $=.98, F(5,995)=2.86, p<.05)$.

El autoconcepto físico presentó relaciones principales con el género, consumo de tabaco, consumo de alcohol y práctica deportiva extraescolar. Respecto al género las diferencias se encontraron en condición física, imagen corporal, competencia percibida, fuerza y autoestima, siendo mayores las puntuaciones obtenidas en los chicos, exceptuando en la dimensión imagen corporal que fue a favor de las chicas. En cuanto al consumo de tabaco se han encontrado diferencias significativas en condición física, imagen corporal, competencia percibida y fuerza, siendo mayores las puntuaciones obtenidas en los que no consumían tabaco, exceptuando en la dimensión imagen corporal que fue a favor de los que fumaban. En la variable consumo de alcohol se han encontrado diferencias significativas solamente en la dimensión imagen corporal, siendo las puntuaciones mayores en los que no consumían alcohol. Cuando se analizó la práctica deportiva extraescolar se encontraron diferencias significativas en las dimensiones imagen corporal, fuerza y autoestima, siendo las puntuaciones mayores para los que practicaban, exceptuando en la dimensión imagen corporal en la que los no practicantes obtuvieron mayores puntuaciones.
Tabla 2

Análisis Univariante y Multivariante de los Factores del Autoconcepto Físico Según el Género, Consumo de Tabaco y Alcohol y Práctica Deportiva.

\begin{tabular}{|c|c|c|c|c|c|}
\hline & \multicolumn{4}{|c|}{ Principales efectos } & \multirow{2}{*}{$\begin{array}{c}\begin{array}{c}\text { Efectos de la } \\
\text { interacción }\end{array} \\
\text { Alcohol x } \\
\text { práctica } \\
\text { deportiva }\end{array}$} \\
\hline & Género & Tabaco & Alcohol & $\begin{array}{l}\text { Práctica } \\
\text { deportiva }\end{array}$ & \\
\hline & $F$ & $F$ & $F$ & $F$ & $F$ \\
\hline Condición física & $1574.65^{* *}$ & $8.31^{*}$ & 2.58 & $20.15^{* *}$ & .15 \\
\hline Imagen corporal & $957.41^{* *}$ & $11.87^{*}$ & $11.54^{*}$ & $33.82^{* *}$ & $8.53^{*}$ \\
\hline $\begin{array}{l}\text { Competencia } \\
\text { percibida }\end{array}$ & $1194.25^{* *}$ & $8.18^{*}$ & .72 & .00 & .06 \\
\hline Fuerza & $918.56^{* *}$ & $4.44^{*}$ & .47 & $6.52^{*}$ & .61 \\
\hline Autoestima & $977.40^{* *}$ & 2.98 & .12 & $13.88^{* *}$ & .61 \\
\hline \multicolumn{6}{|c|}{ Análisis multivariado } \\
\hline Lambda de Wilks & .31 & .98 & .98 & .94 & .98 \\
\hline F multivariado & $431.63^{* *}$ & $3.22^{*}$ & $3.00^{*}$ & $10.87^{* *}$ & $2.86^{*}$ \\
\hline
\end{tabular}

${ }^{*} p<.01 ;{ }^{* *} p<.001$

Tabla 3

Medias y DT del Autoconcepto Físico Según el Género, Tabaco, Alcohol y la Práctica Deportiva.

\begin{tabular}{|c|c|c|c|c|}
\hline \multirow[t]{2}{*}{ Género } & \multicolumn{2}{|c|}{$\begin{array}{c}\text { Chicos } \\
(n=442)\end{array}$} & \multicolumn{2}{|c|}{$\begin{array}{l}\text { Chicas } \\
(n=566)\end{array}$} \\
\hline & $M$ & $D T$ & $M$ & $D T$ \\
\hline Condición fisica & 3.44 & .59 & 1.91 & .59 \\
\hline Imagen corporal & 1.75 & .50 & 2.79 & .62 \\
\hline Competencia percibida & 3.16 & .60 & 1.87 & .47 \\
\hline Fuerza & 3.03 & .59 & 1.84 & .58 \\
\hline Autoestima & 3.37 & .57 & 2.14 & .67 \\
\hline \multirow[t]{2}{*}{ Tabaco } & \multicolumn{2}{|c|}{$\begin{array}{l}\text { No fuma } \\
(n=818)\end{array}$} & \multicolumn{2}{|c|}{$\begin{array}{c}\text { Fuma } \\
(n=190)\end{array}$} \\
\hline & M & $D T$ & M & $D T$ \\
\hline Condición fisica & 2.62 & .95 & 2.43 & .96 \\
\hline Imagen corporal & 2.31 & .76 & 2.47 & .82 \\
\hline Competencia percibida & 2.47 & .83 & 2.31 & .81 \\
\hline Fuerza & 2.40 & .84 & 2.23 & .77 \\
\hline Autoestima & 2.70 & .88 & 2.57 & .81 \\
\hline \multirow[t]{2}{*}{ Alcohol } & \multicolumn{2}{|c|}{$\begin{array}{l}\text { No bebe } \\
(n=502)\end{array}$} & \multicolumn{2}{|c|}{$\begin{array}{c}\text { Bebe } \\
(n=506)\end{array}$} \\
\hline & M & $D T$ & $M$ & $D T$ \\
\hline Condición fisica & 2.57 & .96 & 2.59 & .95 \\
\hline Imagen corporal & 2.38 & .78 & 2.29 & .76 \\
\hline Competencia percibida & 2.44 & .82 & 2.44 & .84 \\
\hline Fuerza & 2.41 & .83 & 2.33 & .83 \\
\hline Autoestima & 2.65 & .89 & 2.70 & .85 \\
\hline \multirow[t]{2}{*}{ Práctica deportiva } & \multicolumn{2}{|c|}{$\begin{array}{c}\text { No practica } \\
(n=394)\end{array}$} & \multicolumn{2}{|c|}{$\begin{array}{l}\text { Practica } \\
(n=614)\end{array}$} \\
\hline & $M$ & $D T$ & $M$ & $D T$ \\
\hline Condición fisica & 2.30 & .46 & 2.76 & 1.14 \\
\hline Imagen corporal & 2.43 & .40 & 2.28 & .93 \\
\hline Competencia percibida & 2.12 & .43 & 2.64 & .95 \\
\hline Fuerza & 2.12 & .55 & 2.52 & .93 \\
\hline Autoestima & 2.50 & .45 & 2.79 & 1.04 \\
\hline
\end{tabular}




\section{Regresión lineal}

Para comprobar la importancia del valor predictivo del género, consumo de tabaco y alcohol y práctica físicodeportiva sobre el autoconcepto físico, se han realizado diferentes análisis de regresión lineal (Tabla 4).

Respecto al poder de predicción del género, el consumo de tabaco y alcohol y la práctica deportiva extraescolar sobre el autoconcepto físico se observó que el ser chico predijo de forma significativa la condición física y muy débilmente la competencia percibida y la fuerza, mientras que ser mujer predijo muy débilmente la imagen corporal. El no consumir alcohol predijo, aunque pobremente, las dimensiones imagen corporal y la fuerza. Por otro lado, la práctica deportiva se mostró como el mejor predictor de la competencia percibida, imagen corporal, condición física y autoestima.

\section{Tabla 4}

Correlaciones, Pesos Beta Estandarizados y Varianza Única y Total para el Género, Consumo de Tabaco, Consumo de Alcohol y Práctica Deportiva como Predictores del Autoconcepto Físico.

\begin{tabular}{lccccccccccccccc}
\hline Variables & $\begin{array}{c}\text { Género } \\
\text { (Chico-chica) }\end{array}$ & \multicolumn{2}{c}{$\begin{array}{c}\text { Fumar } \\
\text { (No fuma- fuma) }\end{array}$} & $\begin{array}{c}\text { Beber } \\
\text { (No bebe-bebe) }\end{array}$ & $\begin{array}{c}\text { Práctica deportiva } \\
\text { (No practica- } \\
\text { practica) }\end{array}$ & $\begin{array}{c}\text { Total } \\
\Delta R^{2}\end{array}$ \\
\hline & $r$ & $\mathrm{~B}$ & $R^{2}$ & $r$ & $\mathrm{~B}$ & $R^{2}$ & $r$ & $\mathrm{~B}$ & $R^{2}$ & $r$ & $\mathrm{~B}$ & $R^{2}$ \\
\hline $\begin{array}{l}\text { Condición } \\
\text { fisica }\end{array}$ & $-.78^{* *}$ & -.32 & $.617^{* *}$ & $-.07^{*}$ & -.01 & .006 & .00 & -.02 & .000 & $.23^{* *}$ & .12 & $.053^{*}$ & .676 \\
$\begin{array}{l}\text { Imagen } \\
\text { corporal }\end{array}$ & $.66^{* *}$ & .09 & $.054^{*}$ & $.08^{* *}$ & .05 & .007 & -.05 & -.12 & $.004^{*}$ & $-.09^{* *}$ & .24 & $.008^{* *}$ & .073 \\
$\begin{array}{l}\text { Competencia } \\
\text { percibida }\end{array}$ & $-.76^{* *}$ & -.28 & $.012^{* *}$ & $-.07^{*}$ & -.02 & .006 & -.00 & -.01 & .000 & $.30^{* *}$ & .41 & $.094^{* *}$ & .112 \\
Fuerza & $-.70^{* *}$ & -.15 & $.005^{* *}$ & $-.07^{*}$ & -.04 & .006 & -.04 & -.15 & $.002^{*}$ & $.23^{* *}$ & .07 & .054 & .067 \\
Autoestima & $-.69^{* *}$ & -.05 & .000 & -.05 & .03 & .003 & .02 & .07 & .001 & $.16^{* *}$ & .13 & $.026^{*}$ & .030 \\
\hline
\end{tabular}

Nota. Se realizaron análisis separados para cada factor. Se presentan las correlaciones $(r)$, los pesos de beta estandarizados (B) y la varianza única $\left(R^{2}\right)$ junto a la varianza total (Total $\left.\Delta R^{2}\right)$.

${ }^{*} p<.01 i^{* *} p<.001$

\section{DISCUSIÓN}

Este estudio ha presentado la relación del autoconcepto físico con las conductas de consumo de tabaco y alcohol y con el género y la práctica deportiva extraescolar en estudiantes adolescentes españoles. Los resultados obtenidos en este estudio parecen apoyar la idea de que el autoconcepto físico presenta relación con las conductas de consumo de tabaco y alcohol además de con el género y la práctica deportiva extraescolar. De esta manera, las chicas son las que tienen un mayor grado de exigencia con su imagen corporal presentando a su vez un autoconcepto más elevado en las fumadoras que no practican actividad físico-deportiva porque según éstas el tabaco ayuda a mantenerse más delgada. Mientras que autores como Jiménez et al. (2008) ofrecen datos contrapuestos que muestran una escasa relación de la autoestima y el autoconcepto de los adolescentes con el consumo de tabaco y alcohol. Sin embargo, existe una relación directamente proporcional entre la elevada cantidad de consumo de dichas sustancias y el alto grado de autoconcepto que presentan los adolescentes (Oliva, Parra, y Sánchez-Queija, 2008). Así lo indican también estudios llevados a cabo en EE.UU. (Shedler y Block, 1990) cuyos resultados se contraponen a los realizados en España por autores como Inglés, Delgado, Bautista, Torregrosa, Espada, García-Fernández et al. (2007) y Moreno, Muñoz, Pérez y Sánchez-Queija (2005), aunque estos últimos matizan la edad de consumo indicando que su práctica en la adolescencia temprana no está relacionada con los problemas de comportamiento y el autoconcepto.

Respecto al género, se encuentran diferencias en todos los parámetros del autoconcepto físico a favor de los chicos, salvo la imagen corporal que es mejor en las chicas, coincidiendo con la idea de autores como De Gracia et al. (2007) que indican que éstas son más críticas con su cuerpo y se preocupan más por la apariencia física que los hombres de modo que si estas detectan una imagen corporal positiva sobre sí mismas mantendrán un autoconcepto físico positivo, mientras que si este parámetro es negativo tendrán que poner en marcha mecanismos que modifiquen esta situación recurriendo a la práctica de actividad físico-deportiva para lograr una mejora en su autoconcepto físico. Mientras, el consumo de tabaco también se relaciona, débilmente con el autoconcepto, con puntuaciones más altas en todos sus factores entre los no fumadores, matizando que la imagen corporal es mejorw entre los fumadores. Respecto al consumo de alcohol, solamente se hallan diferencias en la imagen corporal, obteniendo mayores puntuaciones los no bebedores, en contraposición con Brendgen et al. (2004) quienes afirmaban que un autoconcepto muy elevado en determinadas dimensiones (social y física) indicaba un mayor riesgo de implicación en problemas de carácter externalizante. Otros autores, en cambio, concluyen que ello constituye una protección frente a este tipo de conductas de riesgo (Wild, Flisher, Bahna, y Lombard, 2004). También la práctica deportiva extraescolar se relaciona con mejores puntuaciones en el autoconcepto físico en la mayoría de sus subdominios (a excepción de la imagen corporal que puntúa más alto entre los no practicantes). Este resultado es contrario a otros estudios (Ahmed, Hilton, y Pituch, 2002; Kirkcaldy, Shephard, y Siefen, 2002; Hausenblas y Symons, 2001; Perry et al., 2002) en los que además de fomentar conductas de salud como la práctica de actividad físico-deportiva, aspecto que se relaciona positivamente con el autoconcepto físico, se observa menor presencia en el consumo de alcohol y tabaco. Por el contrario, neumólogos del Departamento de Investigación en Tabaquismo y Enfermedad Pulmonar Obstructiva Crónica del Instituto Nacional de Enfermedades Repiratorias de México, aseguran que dicha práctica no influye en la decisión de reducción del hábito de fumar y que no contrarresta sus efectos sino que su combinación potencia riesgos cardiorrespiratorios. Referente a su poder de predicción, como muestra el análisis de regresión lineal, el ser chico predice mayores puntuaciones en todos los parámetros del autoconcepto físi- 
co, excepto que el ser mujer predice una mejor imagen corporal. El no consumir alcohol predice, muy pobremente, una mejor imagen corporal y la práctica deportiva extraescolar predice también el autoconcepto físico. Algunos autores han mostrado también que la actividad deportiva extraescolar parece ser predictora negativa del consumo de tabaco (Jiménez, Cervelló, García-Calvo, Santos-Rosa, y Del Villar, 2007). En este sentido, en opinión de Jané, Pardell, Saltó y Salleras (2001), la incorporación de la mujer al hábito tabáquico puede estar provocada por el hecho de que el tabaco se relaciona más con aspectos como la autoestima, el estrés, la preocupación por la propia imagen y, sobre todo, el control del peso corporal. Las mujeres están más preocupadas por su peso que los hombres, y para las mujeres jóvenes la imagen corporal es muy importante en estas edades (Thogersen-Ntoumani y Ntoumanis, 2007). Prueba fehaciente de esta circunstancia se encuentra en el estudio sobre el consumo de tabaco en mujeres escolares realizado por Crisp, Stavrakaki, Halek, Williams, Segwick y Kiosiss (1998), donde la ansiedad relacionada con el control del cuerpo, el miedo a sentirse demasiado gordas y a perder el control sobre la ingesta, eran factores que condicionaban el mantenimiento del consumo de tabaco entre las mujeres adolescentes. Así mismo, 0'Loughlin, Paradis, Renaud y Sanchez (1998) y Tomeo, Field, Berkey, Colditz y Frazier (1999) encontraron en mujeres adolescentes que se utilizaba el tabaco como elemento para controlar su peso.

De esta manera, los varones suelen realizar práctica deportiva bajo intereses centrados en la competición deportiva, la exaltación del ego, la aprobación social o la mejora de la condición física. Por ello, es frecuente que los contextos de práctica deportiva en varones sean de naturaleza competitiva y centrados en la práctica deportiva reglada, donde la presencia del tabaco suele ser inexistente. Por el contrario, los motivos de práctica físico-deportiva en las mujeres adolescentes difieren en gran medida de los varones, estando orientados fundamentalmente al cuidado de la estética, la diversión y el establecimiento de relaciones afectivo-sociales (Cechini, Méndez, y Muñiz, 2003; Moreno y Cervelló, 2005). Es importante resaltar que el Observatorio Español sobre Drogas (2004) mantiene que el consumo moderado u ocasional de sustancias es una conducta social llevada a cabo durante la adolescencia y considerada como un acto relativamente normativo en el contexto cultural español.

Este estudio está sujeto a diversas limitaciones que a continuación se detallan. Por un lado, la variable edad, de la que, aunque puede resultar muy reveladora para esclarecer el momento y la intensidad del consumo de sustancias como tabaco y alcohol no se tiene en cuenta su amplitud, considerando sólo la adolescencia con una media de edad de 15.94. De este modo no puede ser relacionada con las demás variables. Por otro lado, la posible contaminación de los datos obtenidos respecto a la información sobre el consumo de sustancias de los adolescentes, aunque investigaciones recientes destacan la fiabilidad proporcionada por estos a la hora de responder los ítems mostrados (Flisher, Evans, Muller, y Lombard, 2004; Sutherland y Shepherd, 2001; Zapert, Snow, y Kraemer, 2002). Finalmente, los resultados obtenidos en este estudio resultan interesantes desde el punto de vista de la relación entre el autoconcepto físico y el género, práctica deportiva extraescolar y consumo de sustancias en la adolescencia, sirviendo como precedente, junto a otros estudios, para intervenir experimentalmente en grupos específicos e intentar comprobar el efecto de la práctica físicodeportiva en la regulación de dichas conductas de consumo y su consecuente mejora en el autoconcepto físico.

\section{REFERENCIAS}

Ahmed, C., Hilton, W., y Pituch, K. (2002). Relationship of strength training to body image among a sample of female university students. Journal of Strength and Conditioning Research, 16, 645-648.

Asçi, F. H., Asçi, A., y Zorba, E. (1999). Cross-cultural validity and reliability of Physical Self-Perception Profile. Journal of Sport and Exercise Psychology, 30, 399-406.

Azorín, F., y Sánchez-Crespo, J. L. (1986). Métodos y aplicaciones del muestreo. Madrid: Alianza Universidad.

Bane, S., y McAuley, E. (1998). Body image and exercise. En J. L. Duda (Ed.), Advances in Sport and Exercise Psychology Measurement (pp. 311-322). Morgantown, WV: Fitness Information Technology.

Bergamaschi, A., Morri, M., Resi, D., Zanetti, F., y Stampi, S. (2002). Tobacco consumption and sports participation: a survey among university students in northern Italy. Annali de Ingieni, 14, 435-442.

Bernaards, C. M., Twisk, J. W., Van Mechelen, W., Snel, J., y Kemper, H. C. (2003). A longitudinal study on smoking in relationship to fitness and heart rate response. Medicine \& Science in Sports \& Exercise, 35, 793-800.

Bertrand, L., y Abernathy, T. J. (1993). Predicting cigarette smoking among adolescents using cross-sectional and longitudinal approaches. Journal of School Health, 63, 98-103.

Brendgen, M., Vitaro, F., Turgeon, L., Poulin, F., y Wanner, B. (2004). Is there a dark side of positive illusions? Overestimation of social competence and subsequent adjustment in aggressive and nonaggressive children. Journal of Abnormal Child Psychology, 32, 305-320.

Bruya, L. D. (1977). Effect of selected movement skills on positive self-concept. Perceptual and Motor Skills, 45, 252-254.

Calleja, N., y Aguilar, J. (2008). Por qué fuman las adolescentes: Un modelo estructural de la intención de fumar. Adicciones, 20, 387-394.

Cecchini, J. A., Méndez, A., y Muñiz, J. (2003). Tendencias o direcciones del deporte contemporáneo en función de los motivos de práctica. Apunts. Educación Física y Deportes, 72, 6-13.

Colbert, L. H., Hartman, T. J., Tangrea, J. A., Pietinen, P., Virtamo, J., Taylor, P. R., y Albanes, D. (2002). Physical activity and lung cancer risk in male smokers. International Journal of Cancer, 98, 770-773.

Crisp, A. H., Stavrakaki, C., Halek, C., Williams, E., Segwick, P., y Kiosiss, I. (1998). Smoking and pursuit of thinness 
in schoolgirls in London and Ottawa. Postgraduate. Medical Journal, 74, 473-479.

De Gracia, M., Marcó, M., y Trujado, P. (2007). Factores asociados a la conducta alimentaria en preadolescentes. Psicothema, 19, 646-653.

Flisher, A. J., Evans, J., Muller, M., y Lombard, C. (2004). Brief report: Test-retest reliability of self-reported adolescent risk behaviour. Journal of Adolescence, 27, 207-212.

Fox, K. R. (1990). The physical self-perception profile. Manual: Northern Illinois University.

Fox, K. R. (1997). The physical self and processes in selfesteem development. En K. R. Fox (Ed.), The physical self: from motivation to well-being (pp. 111-139). Champaign, IL: Human Kinetics.

Fox, K. R., y Corbin, C. B. (1989). The physical self-perception profile: development and preliminary validation. Journal of Sport and Exercise Psychology, 11, 408-430.

Hair, J. F., Anderson, R. E., Tatham, R. L., y Black, W. C. (1998). Multivariate data analysis. Upper Saddle River, New Jersey: Prentice-Hall.

Harter, S. (1986). Processes underlying the construction, maintenance, and enhancement of the self-concept in children. En J. Suls y A. G. Greenwald (Eds.), Psychological perspectives on the self (vol.3, pp. 137181). Hillsdale, NJ: Lawrence Erlbaum.

Harter, S. (1993). Causes and consequences of low self-esteem in children and adolescents. En R. B. Baumeister (Ed.), Self-esteem: The puzzle of low selfesteem (pp. 57-78). New York: Plenum Press.

Hausenblas, H. A., y Symons, D. (2001). Comparison of body image between athletes and nonathletes: A metaanalytic review. Journal of Applied Sport Psychology, 13, 323-339.

Hayes, S. D., Crocker P. R. E., y Kowalski K. C. (1999). Gender differences in physical self-perceptions, global self-esteem, and physical activity: evaluation of the Physical Self-Perception Profile model. Journal of Sport Behaviour, 22, 1-14.

Holmen, T. L., Barrett, E., Clausen, J., Holemn, J., y Bjermer, L. (2002). Physical exercise, sports, and lung function in smoking versus nonsmoking adolescents. European Respiration Journal, 19, 8-15.

Huenemann, R. L., Shapiro, L. R., Hampton, M. C., y Mitchell, B. W. (1966). A longitudinal study of gross body composition and body conformation and their association with food and activity in teenage population. American Journal of Clinical Nutrition, 18, 325-338.

Inglés, C., Delgado, B., Bautista, R., Torregrosa, M., Espada, J., García-Fernández, J., Hidalgo, M., y García-López, L. (2007). Factores psicosociales relacionados con el consumo de alcohol y tabaco en adolescentes españoles. International Journal of Clinical and Health Psychology, 7, 403-420.

Instituto Nacional de Enfermedades Repiratorias de México (2008). El ejercicio no contrarresta los efectos de fumar. México: Departamento de Investigación en Tabaquismo y Enfermedad Pulmonar Obstructiva Crónica.
Jané, M., Pardell, H., Saltó, E., y Salleras, L. (2001). Epidemiología del tabaquismo femenino. Factores determinantes de la iniciación y del mantenimiento. Prevención del Tabaquismo, 3, 147-154.

Jiménez, R., Cervelló, E., M., García, T., Santos-Rosa, F. J., y Iglesias, D. (2007). Estudio de las relaciones entre motivación, práctica deportiva extraescolar y hábitos alimenticios y de descanso en estudiantes de educación física. International Journal of Clinical and Health Psychology, 7, 385-401.

Jiménez, T., Musitu, G., y Murgui, S. (2008). Funcionamiento familiar y consumo de sustancias en adolescentes: el rol mediador de la autoestima. International Journal of Clinical and Health Psychology, 8, 139-151.

Kirkcaldy, B. D., Shephard, R. J., y Siefen, R. G. (2002). The relationship between physical activity and self-image and problem behaviour among adolescents. Social Psychiatry and Psychiatric Epidemiology, 37, 544-550.

Lau, P. W. C., Cheung, M. W. L., y Ransdell, L. B. (2008). A structural equation model of the relationship between body perception and self-esteem: Global physical selfconcept as the mediator. Psychology of Sport and Exercise, 9, 493-509.

Lindwall, M., y Hassmén, P. (2004). The role of exercise and gender for physical self-perceptions and importance ratings in Swedish university students. Scandinavian Journal of Medicine \& Science in Sport, 14, 373-380.

Loland, N. W. (1998). Body image and physical activity. A survey among Norwegian men and women. International Journal of Sport and Exercise Psychology, 29, 339-365.

Maïano, C., Ninot, G., y Bilard, J. (2004). Age and gender effects on global self-esteem and physical selfperception in adolescents. European Physical Education Review, 10, 53-69.

McGowan, R. W., Jarman, B. O., y Pedersen, D. M. (1974). Effects of a competitive endurance-training program on self-concept and peer approval. Journal of Sport Psychology, 86, 57-60.

Melnick, M. J., Miller, K. E., Sabo, D. F., Farrell, M. P., y Barnes, G. M. (2001). Tobacco use among high school athletes and nonathletes: results of the 1997 youth risk behavior survey. Adolescence, 36, 727-747.

Mendoza, R. y López Pérez, P. (2007). El consumo de tabaco en el alumnado español pre-adolescente y adolescente: diferencias de género. Adicciones, 19, 341-356.

Moreno, C., Muñoz, V., Pérez, P., y Sánchez-Queija, I. (2005). Los adolescentes españoles y su salud. Resumen del estudio "Health Behaviour in School Aged Children (HBSC-2002)." Madrid: Ministerio de Sanidad y Consumo.

Moreno, J. A., Cervelló, E., y Moreno, R. (2008). Importancia de la práctica físico-deportiva y del género en el autoconcepto físico de los 9 a los 23 años. International Journal of Clinical, and Health Psychology, 8, 171-183.

Moreno, J. A., y Cervelló, E. (2005). Physical self-perception in Spanish adolescents: effects of gender and involvement in physical activity. Journal of Human Movement Studies, 48, 291-311. 
Nistal, P., Prieto, J. A., Del Valle, M., y González, V. (2003). Relación de la actividad física con el consumo de tabaco en adolescentes. Archivos de Medicina del Deporte, XX(97), 397-403.

Nunnally, J. C. (1978). Psychometric theory. New York: McGraw-Hill.

O’Loughlin, J., Paradis, G., Renaud, L., y Sanchez, L. (1998). One-year predictors of smoking initiation and continued smoking among elementary schoolchildren in multiethnic, low-income, inner-city neighbourhoods. Tobacco Control, 7, 268-275.

Observatorio Español sobre Drogas (2004). Encuesta sobre drogas a población escolar. Madrid: Ministerio del Interior.

Oliva, A., Parra, A., y Sánchez-Queija, I. (2008). Consumo de sustancias durante la adolescencia: trayectorias evolutivas y consecuencias para el ajuste psicológico. International Journal of Clinical and Health Psychology, 8, 153-169.

Packa, B., Massamba, A., Mabiala, J. R., Oniangue, R., Bahanguila, B. A., Gnama, G., Nkoua, J. L., Messanvi, G., y Senga, P. (2002). Recovery from exercise in trained smokers. Annales de Cardiologie et d'Angeiologie, 51, 327-335.

Perry, A. C., Rosenblatt, E. S., Kempner, L., Feldman, B. B., Paolercio, M. A., y Van Bemden, A. L. (2002). The effects of an exercise physiology program on physical fitness variables, body satisfaction, and physiology knowledge. Journal of Strength and Conditioning Research, 16, 219-226.

Shavelson, R. J., Hubner, J. J., y Stanton, G. C. (1976). Validation of construct interpretations. Review of Educational Research, 46, 407-441.

Shedler, J., y Block, J. (1990). Adolescent drug use and psychological health: A longitudinal study. American Psychologist, 45, 612-630.

Shephard, R. J. (1995). Physical Activity, Fitness, and Health: The Current Consensus. Quest, 47, 288-303.
Smith, B. L., Handley, P., y Eldredge, D. A. (1998). Sex differences in exercise motivation and body image satisfaction among college students. Perceptual and Motor Skills, 86, 723-732.

Sonströem, R. J., Speliotis, E. D., y Fava, J. L. (1992). Perceived physical competence in adults: an examination of the physical self-perception profile. Journal of Sports and Exercise Psychology, 14, 207221.

Sutherland, I., y Shepherd, J. P. (2001). Social dimensions of adolescent substance use. Addiction, 96, 445-458.

Teixeira, P. J., Sardinha, L. B., Going, S. B., y Lohman, T. G. (2001). Total and regional fat and serum cardiovascular disease risk factors in lean and obese children and adolescents. Obesity Research, 9, 432-442.

Tercedor, P., Martin-Matillas, M., Chillón, P., Pérez López, I. J., Ortega, F. B., Warnberg J., et al. (2007). Increase in cigarette smoking and decrease in the level of physical activity among Spanish adolescents. AVENA study. Nutrición Hospitalaria, 22, 89-94.

Thogersen-Ntoumani, C., y Ntoumanis, N. (2007). A selfdetermination theory approach to the study of body image concerns, self-presentation and self-perceptions in a sample of aerobic instructors. Journal of Health Psychology, 12, 301-315.

Tomeo, C. A., Field, A. E., Berkey, C. S., Colditz, G. A., y Frazier, A. L. (1999). Weight concerns, weight control behaviors, and smoking initiation. Pediatrics, 104, 918-924.

Wild, L. G., Flisher, A. J., Bhana, A., y Lombard, C. (2004). Associations among adolescent risk behaviours and self-esteem in six domains. Journal of Child Psychology and Psychiatry, 45, 1454-1467.

Zapert, K., Snow, D. L., y Kraemer, J. (2002). Patterns of substance use in early through late adolescence. American Journal of Community Psychology, 30, 835852. 